\title{
Hybrid Ant Colony Optimization-Based Method for Focal of a Disease Segmentation in Lung CT Images
}

\author{
Mingli Lu ${ }^{1(\otimes)}$, Benlian Xu ${ }^{2}$, Weijian Qin ${ }^{1}$, and Jian Shi ${ }^{1,2}$ \\ 1 School of Electrical and Automatic Engineering, \\ Changshu Institute of Technology, Changshu 215500, China \\ lumlecslg.edu.cn \\ 2 School of Mechanical Engineering, \\ Changshu Institute of Technology, Changshu 215500, China
}

\begin{abstract}
The detection of chest CT scan images of the lung play a key role in clinical decision making for some lung disease, such as tumors, pulmonary tuberculosis, solitary pulmonary nodule, lung masses and so on. In this paper, a novel automated CT scan image segmentation algorithm based on hybrid Ant Colony algorithm and snake algorithm is proposed. Firstly, traditional snake algorithm is used to detect the possible edge points of focal of a disease. Then Ant Colony Optimization (ACO) algorithm is applied to search the possible edge points of focal of a disease repeatedly. Finally, real edges can be extracted according to the intensity of pheromones. Simulation experiment results demonstrate that the proposed algorithm is more efficient and effective than the methods we compared it to.
\end{abstract}

Keywords: Ant Colony Optimization · Snake algorithm · Image segmentation · Edge detection

\section{Introduction}

Lung CT scanning is used to detect: tumors in lungs, pneumonia, tuberculosis, emphysema, diffuse interstitial lung diseases, inflammation or other diseases of pleura, the membrane covering the lungs. Automatic identification of lung disorders in lung CT scan images can contribute to lung disease early diagnosis. Edge is the most important features for focal of a disease in CT images, and this feature can be used in target recognition and segmentation. In recent years, research on Segmentation of medicine images has become a hot topic and it has been widely applied in cancer metastasis, developmental biology, immunology response, etc. Conventional and manual analysis of these images is a tedious process. Accuracy Rely on experience and knowledge of observer. However, with the increasing of datasets, manual work is becoming heavy workload and inefficiency. Automated segmentation processing can extract a richness of information far beyond what a manual work can observe. For efficiency and accuracy, the development of automated segmentation methods that eliminate the bias and variability to a 
certain degree is of great importance, which has very broad prospects in clinical decision making.

Because medical images are complex in nature, automatic segmentation of medical images is a challenging task and medical image segmentation continues to be a difficult problem [1]. The challenges of medical image segmentation have been attracting more and more research efforts [2-9]. In [6], Elizabeth et al. proposed an approach to identify the most promising slice to diagnose lung cancer from chest CT images. In [7], an efficient cervical disease diagnosis approach using segmented images and cytology reporting is proposed. In [8], an effective liver vessel segmentation method was proposed based on two techniques, including centerline constraint and intensity model. In [9], a new adaptive approach to lung segmentation based on a non-parametric adaptive active contour method (ACM) is proposed. In summary, from the review presented above, it is found that although some of the segmentation methods mentioned above produce very good segmentation results, the overall performance of the segmentation methods still need to be improved in some situations.

ACO is a population based meta-heuristic approach proposed by Dorigo et al., which is inspired by social behavior of ant colonies and belongs to a branch of swarm intelligence [10]. In nature, ants can find the shortest route between their nest and a food source by chemical materials called pheromone that they leave when moving. Traveling salesman problem (TSP) is the first problem solved by ACO [11]. At present, it is well known that ACO is effective for many optimization problems, such as image processing problems [12], clustering problems [13], vehicle routing problems [14] and resource allocation problem [15].

In the ant colony algorithm for edge detection, searching for interest area is looked upon as an ant colony foraging process. The basic idea applying ACO to edge detection could be explained by a graph, as shown in Fig. 1 .

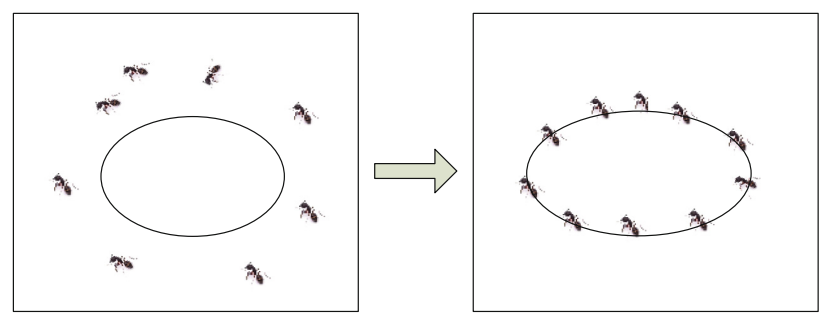

Fig. 1. The evolution process of ant searching for foods

In this paper, motivated by the self-organization ability and positive feedback mechanism of ants, we aim to develop a novel ant-based algorithm for lung CT image segmentation. The remainder of this article is structured as follows. In Sect. 2, the focal of a disease segmentation method is described in details. Section 3 presents the experimental results of the focal zone segmentation. Finally, the fourth section includes the concluding remarks. 


\section{Algorithm}

\subsection{Algorithm Description}

The edge detection of focal zone in lung CT image sequence is very challenging, such as poor signal-to-noise ratios images, intensity inhomogeneity, irregular surfaces and edge branching. As the traditional approach was insufficient for overcome these challenges. So, our goal in this research is to develop a new method for automatic edge extracted in lung CT scan images based on hybrid ant colony algorithm and snake algorithm. The first step in this method is rough edge extracted from lung CT scan image by snake method. Then Ant Colony Optimization (ACO) algorithm is applied to search the possible edge points of focal of a disease, repeatedly. Finally, real edges can be extracted according to the intensity of pheromones. To visualize our proposed algorithm in a full view, we represent the flowchart of the proposed algorithm (Fig. 2).

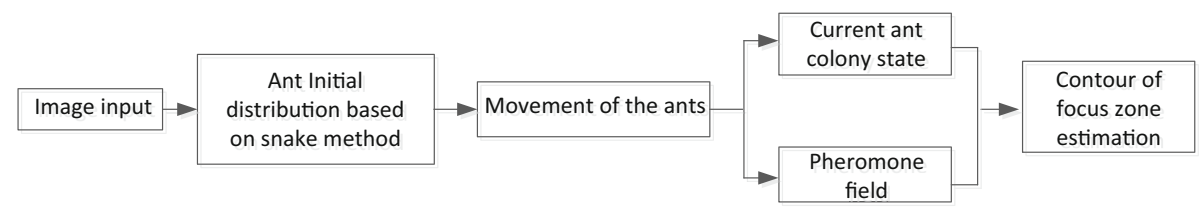

Fig. 2. The main framework of our proposed method

\subsection{Edge Detection Based on Hybrid Ant Colony Algorithm and Snake Algorithm}

Edge is the most important information in CT scan images. Edge characteristics are extracted to clinical decision making for some lung disease. Image edge detection based on hybrid ACO is distributing a certain number of ants on the two dimensional image to search edges by establishing the pheromone matrix, in which each element represents edge information of each pixel. The algorithm contains three steps: Initialization, movement of the ants and pheromone Update.

\subsubsection{Initialization of the Algorithm}

The initialization step is performed at the beginning. A number of ants are randomly assigned on image the possible edge. The initial value of each pheromone matrix component $\tau(0)$ is set to be constant. To help the ACO work faster in finding edge of focal zone, the prior information is utilized to generate initial ant colony. Considered ants are assigned on the rough outline of focal zone, which can be obtained by snake algorithm $[16,17]$.

Snake segmentation algorithm is used to separate the lung tissues from the CT slice by finding a suitable outline. In this method some points are required to initialize the process, the segmentation results are dependent on the choice of outline. Snake segmentation algorithm general consists of drawing curves, starting outside or inside the object of interest. The traditional approach is given as follows. 
Aim to adapt an initial curve to the shape of the region of interest. The curvature occurs by the forces acting on it and evolves to the edges of the object. The deformation is guided by an energy function to be minimized:

$$
B=\sum_{d=0}^{M-1}\left\{B_{\mathrm{int}}[c(d)]+B_{\text {ext }}[c(d)]\right\}
$$

where $c$ is the initial contour curve and $c(d)$ is point on the curve $c, M$ is the total length of $c . B_{\text {int }}[c(d)]$ is the internal energy, which depends on the internal features within the segmentation curve and can be given by

$$
B_{\text {int }}\left[c(d)=\sigma(d) c^{\prime}(d)+v(d) c^{\prime \prime}(d)\right.
$$

where $\sigma$ and $v$ are the parameters to effect the evolution of curvature at a point $d$ of the curve, which can be adjusted according to the first and second derivatives, respectively. At the same time, $B_{\text {ext }}[c(d)]$ represents the external energy of this curve. The environment outside the curve can change this energy. The behavior of the curve, such as expansion or shrinkage is continue guided by an energy function $B$, until it reaching the boundaries of the object of interest.

After rough edge of focal zone is obtained by snake method, ants are assigned on the rough edge of focal zone.

\subsubsection{Movement of the Ants}

In contrast with classic ACO, here, nodes in the graph can be viewed as pixels of the image. During search process, an ant chooses which pixel to move to according to heuristics information and the pheromone amount of the four surrounding pixels. Assume ant $a$ at the current position of pixel $i$, The probability that ant $a$ moves from the pixel $(i)$ to its neighboring pixel $(j)$ is computed by

$$
p_{i j}^{a}(t)= \begin{cases}\frac{\left[\tau_{j}(t)\right]^{\gamma}\left[\eta_{j}\right]^{\beta}}{\sum_{j^{\prime} \in \Omega(i)}\left[\tau_{j^{\prime}}(t)\right]^{\gamma}\left[\eta_{j}\right]^{\beta}}, & \text { if } j \in \Omega(i) \\ 0, & \text { otherwise }\end{cases}
$$

where $p_{i j}^{a}$ is the probability with which ant $a$ chooses to move from the pixel $i$ to the pixel $j$ at the $t$-th iteration. $\Omega(i)$ is the set of all available neighbors of pixel $i, \tau_{j}$ is the pheromone value on pixel $j$ at the $t$-th iteration, and $\eta_{j}$ is heuristic value, for each pixel, usually represents the attractiveness of the pixel. representing the degree of similarity between the current pixel and the target pixel. $\gamma$ and $\beta$ are the weights of the pheromone value and heuristic value, respectively. $\gamma$ determines the relatively importance of the track, reflecting the effect of accumulated information of the ant in the course of movement. $\beta$ makes up the comparative importance of heuristic information.

$\eta_{j}$ is heuristic information of pixel $j$, which is estimated based on gradient information of image.

$$
\eta_{j}=\left(\left|I_{j}^{(x+1, y)}-I_{j}^{(x-1, y)}\right|+\left|I_{j}^{(x-1, y+1)}-I_{j}^{(x+1, y-1)}\right|\right.
$$




$$
\left.+\left|I_{j}^{(x, y+1)}-I_{j}^{(x, y-1)}\right|+\left|I_{j}^{(x+1, y+1)}-I_{j}^{(x-1, y-1)}\right|\right) / I_{\max }
$$

where $I_{j}^{(x, y)}$ denotes intensity level on pixel $j$ with the coordinate $(x, y) . I_{\max }$ is the maximum intensity value of the image.

\subsubsection{Pheromone Updating}

Pheromone is another important concept in ACO algorithms. In this work, we considered two kinds of pheromone, diffusive pheromone and accumulative pheromone. Diffusive pheromone is the propagated information from the different channels of the neighbor pixels. Accumulative pheromone is the accumulative information in each step. When all ants complete a search cycle, pheromone on pixel $j$ is updated according to the following formula:

$$
\tau_{j}(t) \leftarrow(1-\varphi) \tau_{j}(t-1)+\Delta \tau_{j}(t-1)+h_{j}(t-1)
$$

where $\varphi$ is pheromone decay coefficient representing pheromone evaporation $(0<\varphi<$ 1). $\Delta \tau_{j}(t-1)=\sum_{a=1}^{N} \Delta \tau_{j}^{a}$ represents the increment of pheromones in the pixel $j$ in this iteration, $\Delta \tau_{j}^{a}$ is the amount of pheromone left by ant $a$ on pixel $j$. Term $h_{j}(t-1)$ models all diffusion input to pixel $j$.

With the increase of iteration, the search router of the ant gradually converges to the true contour, and the pheromone on the edge is significantly higher than other regions. Once the searching behavior of each ant is finished, thus edge according to the pheromone distribution can be extracted.

\section{Experiments}

In this section, we will discuss the implementation process in detail to verify the validity of our proposed method. All experiments were carried out in MATLAB (R2016a) on a 1.7 $\mathrm{GHz}$ processor computer with $4 \mathrm{G}$ random access memory.

Several human lung CT scans image with different disease, such as pulmonary tuberculosis (frame D0100249, D0100252, D0100263-D0100265), solitary pulmonary nodules (frame D0100151), were selected as the experimental images and the image was $512 \times 512$ pixels. The key parameters are as follows: $N_{\text {ant }}=300$ is the number of ant colony, $N_{\max }=30$ is the number of iteration times, the heuristic factor $\gamma$ and $\beta$ are 0.5 , 1 respectively. The experimental results are shown in Fig. 3 and Fig. 4. Traditional ant colony algorithm and our proposed hybrid segmentation algorithm are compared that uses the same test images.

To evaluate the performance of the proposed approach on segmentation, we would like to compare our algorithm with other techniques, such as the traditional ant colony algorithm [18]. According to Fig. 3 and Fig. 4, the results obtained in this work show that our proposed hybrid segmentation method presents promising potential and has excellent results for accuracy compared to the traditional ant colony algorithm.

In addition, average computation time (over 100 Monte-Carlo simulations) using our proposed method is not exceeding $15 \mathrm{~s}$ for all frames. The average time was shorter 


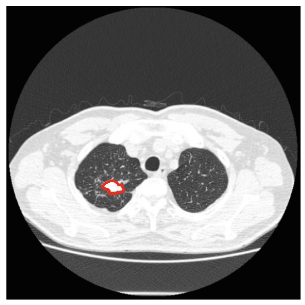

D0100263

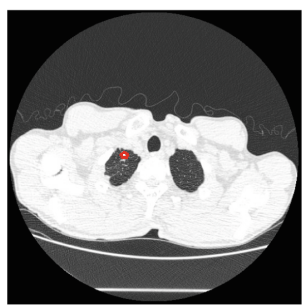

D0100252

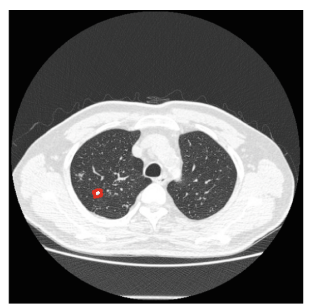

D0100264

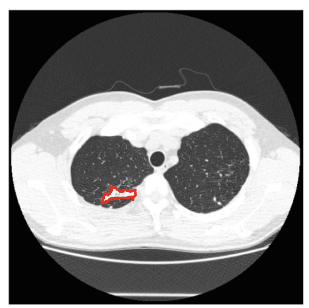

D0100249

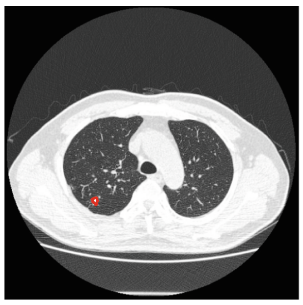

D0100265

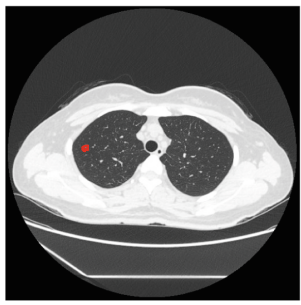

D0100151

(a) Tracking results of original lung CT image

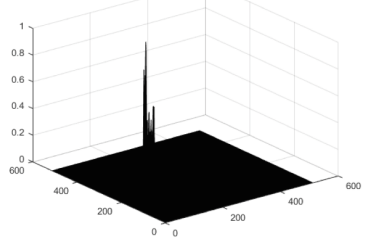

D0100263

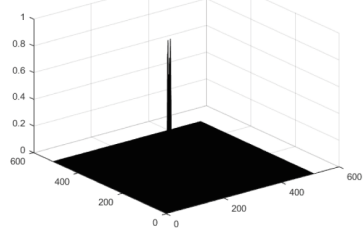

D0100252

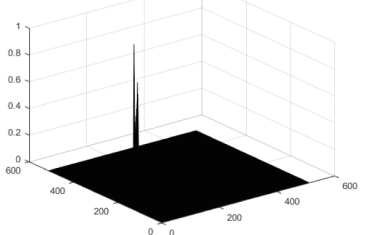

D0100264

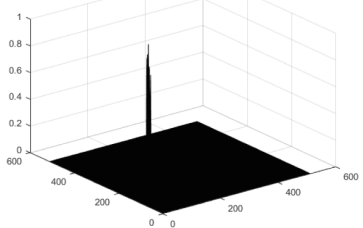

D0100151

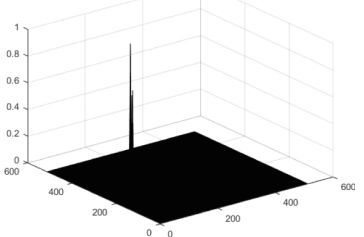

D0100265

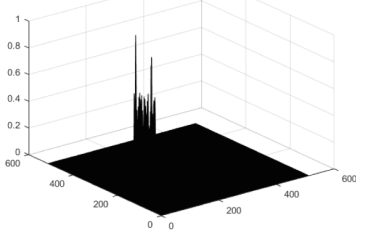

D0100249

(b)The resulting ant pheromone field

Fig. 3. Tracking results with our proposed mode

than traditional ACO. Our proposed approach can identify the region near the focal zone contour of the lung, accelerating the segmentation process. 


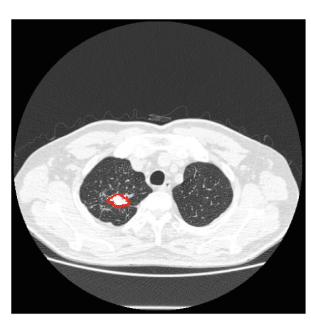

D0100263

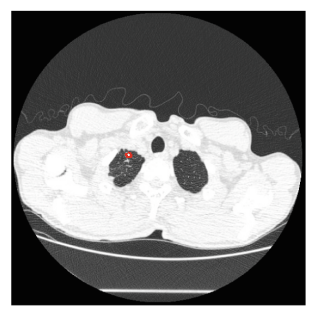

D0100252

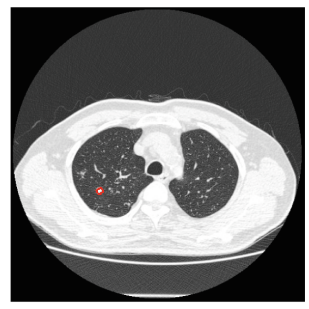

D0100264

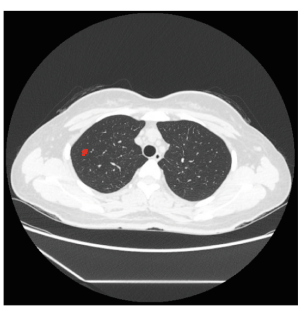

D0100151

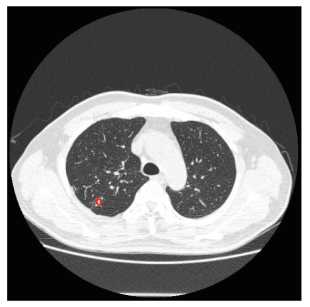

D0100265

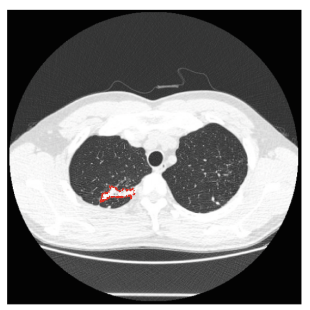

D0100249

Fig. 4. Tracking results with traditional ACO

In summary, from the results, it can be clearly inferred that the proposed hybrid segmentation method has competitive potential compared to the other techniques.

\section{Conclusions}

Lung CT image provide lots of important information for lung-disease diagnosis and lung surgery. In this paper, a hybrid swarm intelligent approach for focal of a disease segmentation lung CT image was proposed. The lung tissues are segmented using our proposed edge detection approach. Edges are extracted from the segmented lung and from which geometrical features are extracted. Experimental results show that proposed method was robust and efficient compared with some traditional methods.

Acknowledgments. This work was supported by National Natural Science Foundation of China (No. 61876024 and No. 61673075), Project of talent peak of six industries (2017-DZXX-001), 333 Project of Jiangsu Province (No. BRA2019284), and partly supported by Jiangsu Laboratory of Lake Environment Remote Sensing Technologies Open Project Fund (JSLERS-2017-006) and The Science and Technology Development Plan Project of Chang Shu (CR0201711).

\section{References}

1. Sharma, N., Aggarwal, L.M.: Automated medical image segmentation techniques. J. Med. Phys. 35(1), 3-14 (2010) 
2. Arnay, R., Fumero, F., Sigut, J.: Ant Colony Optimization-based method for optic cup segmentation in retinal images. Appl. Soft Comput. 52, 409-417 (2017)

3. Chitradevi, D., Prabha, S.: Analysis of brain sub regions using optimization techniques and deep learning method in Alzheimer disease. Appl. Soft Comput. 86, 105857 (2020)

4. Bhattacharjee, K., Pant, M.: Hybrid particle swarm optimization-genetic algorithm trained multi-layer perceptron for classification of human glioma from molecular brain neoplasia data. Cogn. Syst. Res. 58, 173-194 (2019)

5. Yang, X., et al.: Segmentation of liver and vessels from CT images and classification of liver segments for preoperative liver surgical planning in living donor liver transplantation. Comput. Methods Programs Biomed. 158, 41-52 (2018)

6. Elizabeth, D.S., Nehemiah, H.K., Raj, C.S.R., Kannan, A.: Computer-aided diagnosis of lung cancer based on analysis of the significant slice of chest computed tomography image. IET Image Proc. 6, 697-705 (2012)

7. Chen, H., Yang, L., Li, L., Li, M., Chen, Z.: An efficient cervical disease diagnosis approach using segmented images and cytology reporting. Cogn. Syst. Res. 58, 265-277 (2019)

8. Zeng, Y., Zhaoa, Y., Liaoa, S., Liaoc, M., Chend, Y., Liu, X.: Liver vessel segmentation based on centerline constraint and intensity model. Biomed. Signal Process. Control 45, 192-201 (2018)

9. Medeiros, A.G., et al.: A new fast morphological geodesic active contour method for lung CT image segmentation. Measurement 148(1-13), 106687 (2019)

10. Dorigo, M., Maniezzo, V., Colorni, A.: Ant system: optimization by a colony of coop-erating agents. IEEE Trans. Syst. Man Cybern. 26(1), 29-41 (1996)

11. Zhou, Y.: Runtime analysis of an ant colony optimization algorithm for TSP instances. IEEE Trans. Evol. Comput. 13(5), 1083-1092 (2009)

12. Miria, A., Sharifianb, S., Rashidib, S., Ghodsca, M.: Medical image denoising based on 2D discrete cosine transform via ant colony optimization. Optik 156, 938-948 (2018)

13. Abbas, F., Fan, P.: Clustering-based reliable low-latency routing scheme using ACO method for vehicular networks. Veh. Commun. 12, 66-74 (2018)

14. Huanga, S.-H., Huangb, Y.-H., Blazquezc, C.A., Paredes Belmarda, G.: Application of the ant colony optimization in the resolution of the bridge inspection routing problem. Appl. Soft Comput. 65, 443-461 (2018)

15. Wang, X., Choi, T.-M., Liu, H., Yue, X.: Novel ant colony optimization methods for simplifying solution construction in vehicle routing problems. IEEE Trans. Intell. Transp. Syst. 17(11), 3132-3141 (2016)

16. Yang, S.-C., Cheng-Yi, Y., Lin, C.-J., Lin, H.-Y., Lin, C.-Y.: Reconstruction of threedimensional breast-tumor model using multispectral gradient vector flow snake method. $\mathrm{J}$. Appl. Res. Technol. 13, 279-290 (2015)

17. Bessa, J.A., Cortez, P.C., da Silva Félix, J.H., da Rocha Neto, A.R., de Alexandria, A.R.: Radial snakes: comparison of segmentation methods in synthetic noisy images. Expert Syst. Appl. 42, 3079-3088 (2015)

18. Li, L.: SAR image oil film detection based on ant Colony Optimization algorithm, International Congress on Image and Signal Processing. In: Bio-Medical Engineering and Informatics, pp. 619-623 (2016) 